Christley, Y., Hollins Martin, C.J. and Martin, C.R. (2012). Perinatal perspectives on Chronic Fatigue Syndrome. British Journal of Midwifery. 20(6): 389-393.

\author{
Yvonne Christley $^{1}$ \\ Caroline J. Hollins Martin PhD, MPhil, BSc, PGCE, ADM, RM, RGN² \\ Colin R. Martin PhD BSc RMN ${ }^{3}$
}

Address for correspondence: Prof Caroline J. Hollins Martin, Professor in Midwifery, School of Nursing, Midwifery and Social Work, MS 2.78, University of Salford.

E-mail: C.J.Hollins-Martin@salford.ac.uk 


\title{
Perinatal perspectives on Chronic Fatigue Syndrome (CFS)
}

\begin{abstract}
Chronic Fatigue Syndrome (CFS) is a severe, systemic, acquired illness that presents with overpowering tiredness that cannot be relieved by rest and is deteriorated through physical and mental activity. Due to the window of prevalence (20-40 years), some women diagnosed with CFS become pregnant. Given that CFS targets women in their reproductive years, responsibility is placed upon midwives to educate, support and provide families with advice. To equip midwives with knowledge about CFS, the objectives of this paper are to provide an overview of: (1) How CFS impacts on reproductive health, (2) The effects of pregnancy on CFS symptoms, (3) CFS related pregnancy complications, (4) Intrapartum management of women with CFS, and (5) Postpartum recovery of women with CFS. To date, only a handful of studies have explored relationships between CFS, childbearing and associated complications. Greater understandings of CSF interactions with physical, psychological and social reproductive processes are required.
\end{abstract}

Word count - 3, 976 (total article)

Key words: Chronic Fatigue Syndrome (CFS), midwives, obstetric, childbearing women

\section{Keypoints}

(1) Prevalence of CFS is thought to range between $0.004-2.6 \%$ in the general population.

(2) The few studies that have been conducted produce compelling evidence of a positive correlation between CFS and gynecological complications.

(3) $30 \%$ of women with CFS experience improved symptoms during pregnancy, $41 \%$ encounter no change, and $29 \%$ report deterioration. Decline in condition occurs in $50 \%$ of women postpartum.

(4) Women with CFS are vulnerable to developing depressive psychopathology postpartum.

(5) No evidence supports that pregnant woman can directly transmit CFS to their unborn child. 


\section{Perinatal perspectives on Chronic Fatigue Syndrome (CFS)}

\section{Introduction}

According to Christley et al (2010) Chronic Fatigue Syndrome (CFS) is categorized by an assorted range of features. These include the acquirer developing overwhelming and enduring fatigue that is not improved by bed rest. The fatigue is so severe that it compromises the individual's usual daily activity to less than $50 \%$ of their normal capacity for at least 6 months. Potential illnesses which might account for symptoms must be excluded as possible causes. Signs and symptoms include: mild fever, sore throat, tender lymph nodes, muscle weakness, malaise post-exertion, muscle pain, new onset headaches, joint pain and lymphadenopathy. Neuropsychiatric symptoms include: depression, irritability, confusion, thinking problems, memory problems, photophobia and sleep difficulties. These clinical features must have appeared over a few hours or a few days and are persistent over a period of 6 months

Precise cause of CFS remains unspecified and currently there are no tests to facilitate diagnosis. Identification of the condition is based upon clusters of symptoms that are activated by numerous factors (Jason et al, 2011). In the absence of aetiology of cause, diagnosis is based upon exclusion, subjective clinical interpretation and patient reports of the experience (Buchwald et al, 1996).

The prevalence of CFS varies extensively due to lack of agreement in case definition, differences in methods of labeling the condition and situational features (Cho et al, 2009). Prevalence is thought to range between $0.004-2.6 \%$ in the general population (Bazelmans et al, 1999; Njoku et al, 2007; Reeves et al, 2007), depending upon case definitions used. Classically CFS affects young adults, with peak age of onset ranging from 20-40 years (Capelli et al, 2010) and occurring 4 times more often in females over males (Reeves et al, 2007). Substantial physical, social and economic disability accompanies CFS, with related clinical features significantly interfering with the lifestyle of women during peak reproductive years. Due to the window of prevalence (20-40 years), some women diagnosed with CFS become pregnant, with a dearth of literature focusing upon management of the 
condition during the antenatal and postnatal period. Since CFS strikes women in their prime reproductive years, the condition becomes part of the midwives remit. To equip midwives with understandings about CFS, this paper provides an overview of:

- How CFS impacts on reproductive health.

- The effects of pregnancy on CFS symptoms.

- CFS related pregnancy complications.

- Intrapartum management of women with CFS.

- Postpartum recovery of women with CFS.

\section{How CFS impacts on reproductive health}

Given that high rates of CFS afflict women of childbearing age, it is suprising that so few studies have investigated influence upon reproductive health. The few studies conducted produce compelling evidence of a positive correlation between CFS and gynecological complications (Boneva et al, 2011; Hamilton et al, 2009; Harlow et al, 1998; MacDonald et al, 1996; Reyes et al, 1996; Sinaii et al, 2002). For example, Reyes et al. (1996) showed that women with CFS are (51\%) more likely to require a hysterectomy, compared with a matched case control group of women (31\%). This finding is supported by Boneva et al. (2011) who conducted a population-based case control study of gynaecological health that relates to CFS. Boneva et al. (2011) reported that, in addition to higher rates of hysterectomy and oophorectomy, women with CFS are 3 times more likely to experience endometriosis, which is consistent with the findings of Sinaii et al. (2002) and Harlow et al. (1998). On average women with CFS experience menopause 4.4 years earlier (mean age 41.7) than their non CFS counterparts (mean age 46.1). They are also more likely to encounter amenorrhea (Boneva et al, 2011).

Harlow et al (1998) reports that $20 \%$ of women experience endometriosis predating their CFS diagnosis also consistent with the findings of Sinaii et al (2002). Associations between endometriosis, CFS and additional functional somatic syndromes (e.g., fibromyalgia) have led researchers to conclude that these conditions share common, albeit not well understood, pathogenic pathways (Boneva et al, 2011; Harlow et al, 1998; Sinaii et al, 2002; Straus et al, 1988). Women with CFS have an increased risk of 
experiencing menstrual cycle irregularities (Harlow et al., 1998), which manifest with episodes of amenorrhea and intermittent mid cycle bleeding. Polycystic ovarian syndrome, uterine fibroids, ovarian cysts and sexually transmitted diseases are more likely to precede a CFS diagnosis, with antecedent pathophysiology unclear (Harlow et al, 1998).

Whilst literature has unearthed a number of associations between CFS and reproductive anomalies, relationships with fertility are unclear. Tentative correlations are proposed between CFS and reduced number of pregnancies (Hamilton et al, 2009; MacDonald et al, 1996). MacDonald et al (1996) found women with CFS more likely to be nultiparous. In a case control study, (9 out of 18 (50\%) of women with CFS compared with (9 out of 36 (25\%) unaffected women were recorded to be nultiparous. Hamilton et al (2009) produced a report which proposes that women with CFS birth fewer children in the 3 years preceding their CFS diagnosis, in comparison to non TS equivalents. Harlow et al (1998) presents the explanation that endocrine abnormalities blight fertility in women with CFS (Harlow et al, 1998), with relationships between dysmenorrhea and non-ovulatory menstrual cycles potentially caused by ovarian hyperandrogenism and/or hyperprolactinemia. Hypothetically, the immunomodulatory effects of progesterone in the presence of unremitting oestrogen production increases risks of developing CFS like symptoms (Harlow et al, 1998).

To date, a dearth of studies have explored discrepancies between women with CFS seeking to avoid conception, and those simply unable to conceive. Considering the additional strains of pregnancy and motherhood, women with CFS might elect to avoid further encumbrances, augmented by uncertainties about progression of the condition and its limited treatment options. Schacterle and Komaroff (2004) conducted a survey that established that $21 \%$ of women with CFS purposely avoid motherhood because of self perceived limitations in their parenting ability that directly relates to CFS. Quite clearly, further research into identifying relationships between gynecological problems and CFS is required.

\section{Effects of pregnancy on CFS symptoms}

Paucity of research has examined effects of CFS on pregnancy and symptom improvement or deterioration during its course. Schacterle and Komaroff 
(2004) conducted a retrospective self-report investigation of women with CFS $(n=86)$, which explored a cumulative total of 252 pregnancies before and after diagnosis. Findings showed that $30 \%$ of women with CFS experience improved symptoms during pregnancy, $41 \%$ encounter no change, and $29 \%$ report deterioration. A decline in condition was reported by $50 \%$ of postpartum participants (Schacterle and Komaroff, 2004). Literature on autoimmune conditions, e.g., multiple sclerosis (Finkelsztejn et al, 2011) and rheumatoid arthritis (Ostensen and Villiger, 2007), has shown that pregnancy often proceeds with improved symptoms during pregnancy that often radically deteriorate postpartum. One explanation for prenatal reduced symptomology is hormone induced changes to the immune system during pregnancy. Adaption of the maternal immune system to the semi-allergenic fetus is thought to diminish pathology in rheumatoid arthritis and multiple sclerosis (Finkelsztejn et al, 2011). To date, no literature explains improved or deteriorated CFS symptoms during pregnancy, although physiological explanations may bear likeness.

\section{CFS related pregnancy complications}

Schacterle and Komaroff (2004) examined CFS related pregnancy complications. They compared problems that arose during pregnancy in participants before and after CFS development. Rate of spontaneous miscarriage in the first trimester was found to be 4 times greater in women with $\mathrm{CFS}$, with higher numbers requiring dilation and curettage (D\&C) post miscarriage (Boneva et al, 2011). There is also a higher rate of recorded developmental delays and learning disability in offspring of women who have conceived post CFS diagnosis. One criticism of this study is that data may have been skewed by confounding variables, such as mothers' age not being taken into consideration.

There is no recorded negative impact effects of CFS on established pregnancy, specifically in relation to low birth weight, prematurity, neonatal death and pre-eclampsia (Schacterle and Komaroff, 2004). Women with CFS often worry about the impact their condition may have on the future health and wellbeing of their fetus (Schacterle and Kmaroff, 2004). Schacterle and Kmaroff (2004) concluded that around $20 \%$ of women with CFS decide to 
avoid pregnancy through concerns about producing a compromised child. In terms of heritability, existing literature has produced convincing evidence that genetic factors contribute to the development and progression of CFS in adults (Albright et al, 2011; Kaiser, 2011). Twin studies have shown that both genetic and environmental factors contribute to familial clustering's of CFS (Schur et al, 2007), with first-degree relatives having an increased chance of developing CFS compared with non matched controls (Walsh et al, 2001). Albright et al (2011) estimates the risk of first-degree relatives developing CFS to be around $2.7 \%$. Smith et al (2010) identified higher rates of severe fatigue in adolescent offspring of women with CFS compared with those borne of healthy mothers. Further study is warranted to identify environmental variables that influence development of CFS, with the causal picture clearly complex. It appears that genetics play some part and the environment another, in relation to onset and progression of CSF. To date no evidence supports that pregnant woman can directly transmit CFS to their unborn child.

\section{Intrapartum management of women with CFS}

To date, no studies have assessed women with CFS experiences of intranatal events. Of relevance are reports from the wider literature that fatigue can adversely effect labour (Boneva et al, 2011; Chien and Ko, 2004; Mayberry et al, 1999). Chien and Ko (2004) report that women who experience fatigue during pregnancy are less able to cope with pain during labour and are compromised whilst attempting to push during second stage. Together these factors increase the likelihood of a woman with CFS receiving caesarian section or forceps delivery. Mayberry et al (1999) and Boneva et al (2011) recognized that gynecological surgery is more common in women with CFS (44.4\%), in comparison to a matched case control group (25\%). To date, no studies have identified aetiology of biochemical pathways and psychological relationships between fatigue and the childbearing women's ability to perform during labour. Allen (2008) proposes that exposing women with CFS to a prolonged and painful labour may exacerbate postpartum symptoms. Of importance to midwives, is that childbearing women with CFS should be cautiously assessed for their willingness and fitness to labour. If women are to be empowered to make choices for childbirth, it is important that midwives 
explore and discuss their wishes and feelings about the delivery options available. Providing practical and sensible information should facilitate women to construct a more accurate picture from which realistic hopes, fears and expectations can be formulated about impending labour (Hollins Martin, 2008). Since experiences of childbirth differ, it is recommended that midwives audit women with CFS birth satisfaction against their prewritten birth plans. Articulated reflections of birth experience would facilitate understanding of what women with CFS perceive they can cope with. The evidence base would be enlarged through practical studies that investigate such relationships.

\section{Postpartum recovery of women with CFS}

The effect of CFS on postpartum recovery has yet to be investigated in a meaningful way. Pregnancy and childbirth places considerable physical and psychological stress on the healthy childbearing woman, with the CFS experiencer having these customary stressors augmented by their condition. Excessive fatigue in the postnatal period can transform the day-to-day responsibilities of family life into an overwhelming experience (Giallo et al, 2011). Women with CFS already face intense fatigue (Dancey and Friend, 2008), which will be enhanced by exhaustion ordinarily associated with routine transition to motherhood. Postpartum fatigue is just one precursor to developing Post Natal Depression (PND), adjoined by numerous other psychosocial antecedents, e.g., a history of depressive illness, excessive stress, low self-esteem, and/or poor social support (Baldwin and Papakostas, 2006). Outside childbearing, an estimated $50-80 \%$ of CFS experiencers' are diagnosed with reactive depression during the course of their illness (Taillefer et al., 2003). PND is already known to limit a new mother's ability to effectively care for and bond with her baby, and the condition is known to stimulate feelings of isolation, guilt, helplessness and despair (O'Hara, 2009). To complicate the clinical picture, mothers with PND are often less reactive to infant cues and can present with parenting behaviours that engender hostility and disengagement (Field, 2009, 2010). Discord in the maternal-infant relationship is associated with compromised cognitive functioning and dissonant emotional development off offspring (Moses-Kolko and Roth, 2004). Stress from child rearing causes $10-20 \%$ of women with no personal or family 
history to develop mental health problems in the perinatal period (Knightley, 2008). Considering that healthy women are already vulnerable to developing postnatal depressive psychopathology, it is salient that midwives and health visitors screen those with CFS routinely for PND. CFS by virtue of diagnosis creates problems with interpersonal functioning, which will add to the routine stressors in a postnatal environment. Ordinarily, there are known variables that promote or debilitate routine transition to motherhood. For example, Brown and Harris (1978) isolated 4 features that promote mental health disturbance in postnatal woman. When all 4 predictors are present, $100 \%$ of mothers become depressed:

(1) Lack of an intimate relationship.

(2) Three or more children under the age of 14.

(3) Loss of own mother before the age of 11.

(4) No employment outside the home.

Further predictors of psychological disturbance include, the childbearing woman having a poor relationship with her own mother (Murry et al, 1995), her partner (Richards, 1990), and minimal support networks (Richards, 1990). Other factors that influence a positive or negative transition to motherhood include, feelings orientated towards the baby, the infant's sleep pattern, contentedness of the baby, amount of support with household chores, quality of companionships, self esteem, intranatal experience (Hollins Martin, 2012) and birth satisfaction (Hollins Martin and Fleming, 2011).

The Edinburgh Postnatal Depression Scale (EPDS) (Cox, 1986, Cox and Holden, 2003) is an instrument designed to facilitate diagnosis of PND. Since women with CFS are already placed in the "at risk" category, it is important for midwives to assess and screen for PND. In the event of a diagnosis, subsequent steps include following the mental health protocol and commissioning support networks. Educating childbearing woman with CFS, partner and family about how to prevent, diagnose and ameliorate mental health disturbance is an essential component of the midwives role.

\section{Conclusion}

CFS is a severe, systemic, acquired illness that presents with overwhelming fatigue and is exacerbated by physical and mental activity. Aetiology is 
insufficiently understood and at present there are no effective cures. Given that CFS targets women in their reproductive years, responsibility is placed upon midwives to educate, support and provide families with appropriate advice. To date, only a handful of studies have explored relationships between CFS, childbearing and holistic associated complications, with greater understandings of interactions with physical, psychological and social reproductive processes required. Many unanswered research questions remain. For example:

- How does childbearing and rearing exacerbate clinical features of CFS?

- What are women with CFS experiences of pregnancy and childbirth?

- How does having CFS affect childbearing women's transition to motherhood?

- What factors help women with CFS cope better with childbearing?

- How can midwives help women with CFS cope when they receive a PND diagnosis?

Midwives working with childbearing women require to identify those at risk of developing perinatal mental health problems. Woman with CFS clearly stand in the "at risk" group, and therefore the woman and her family should be appropriately psychologically prepared, monitored and supported during pregnancy and the postnatal period, as is the request of the $\mathrm{CEMACH}$ report (2004). 


\section{References}

Albright F, Light K, Light A, Bateman L, Cannon-Albright LA (2011) Evidence for a heritable predisposition to chronic fatigue syndrome. BMC Neurology 11: 62.

Allen PR (2008) Chronic fatigue syndrome: implications for women and their health care providers during the childbearing years. Journal of Midwifery and Women's Health 53(4): 289-99.

Baldwin DS, Papakostas GI (2006) Symptoms of fatigue and sleepiness in major depressive disorder. Journal of Clinical Psychiatry 67(6): 9-15.

Bazelmans E, Vercoulen JH, Swanink CM (1999) Chronic fatigue syndrome and primary fibromyalgia syndrome as recognized by GPs. Family Practitioner. 6(6): 602-604.

Boneva RS, Maloney EM, Lin JM (2011) Gynecological history in chronic fatigue syndrome: a population-based case<en>control study. Journal of Women's Health 20(1), 21-28.

Brown G, Harris T (1978) The social origins of depression. Tavistock, London.

Buchwald D, Pearlman T, Umali J, Schmaling K, Katon W (1996) Functional status in patients with chronic fatigue syndrome, other fatiguing illnesses, and healthy individuals. American Journal of Medicine 101(4): 364-370.

Capelli E, Zola R, Lorusso L, Venturini L, Sardi F, Ricevuti G (2010) Chronic fatigue syndrome/myalgic encephalomyelitis: an update. International Journal of Immunopathology and Pharmacology 23(4): 981-989.

Chien LY, Ko YL (2004). Fatigue during pregnancy predicts caesarean deliveries. Journal of Advanced Nursing 45(5): 487-494.

Cho HJ, Menezes PR, Hotopf M, Bhugra D, Wessely S (2009) Comparative epidemiology of chronic fatigue syndrome in Brazilian and British primary care: prevalence and recognition. British Journal of Psychiatry 194, 117-122.

Christley Y, Duffy T, Martin CR (2010) A review of the definitional criteria for chronic fatigue syndrome. Journal of Evaluation in Clinical Practice 18(2012: 25-31.

Cox J (1986) Postnatal Depression: a guide for health professionals. Churchill Livingston, Edinburgh.

Confidential Enquiries into Maternal and Child Health (CEMACH) (2004) Why mothers die: report of the confidential enquiries into maternal deaths in the UK 2000-2002. RCOG Press, London.

Cox J, Holden J (2003) Perinatal mental health: a guide to the Edinburgh 
Postnatal Depression Scale. Royal College of Psychiatrists, London.

Dancey CP, Friend J (2008) Symptoms, impairment and illness intrusiveness. Their relationship with depression in women with CFS/ME. Psychology and Health 23(8): 983-99.

Field T (2010) Postpartum depression: effects on early interactions, parenting, and safety practices: a review. Infant Behaviour and Development 33(1): 1-6.

Finkelsztejn A, Brooks JB, Paschoal FM, Fragoso YD (2011) What can we really tell women with multiple sclerosis regarding pregnancy: a systematic review and meta-analysis of the literature. BJOG an International Journal of Obstetrics and Gynaecology 118(7): 790-797.

Giallo R, Wade C, Cooklin A, Rose N (2011) Assessment of maternal fatigue and depression in the postpartum period: support for two separate constructs. Journal of Reproductive and Infant Psychology 29(1): 69-80.

Hamilton WT, Gallagher AM, Thomas JM, White PD (2009) Risk markers for both chronic fatigue and irritable bowel syndromes: a prospective case<en>control study in primary care. Psychological Medicine 39(11): 19131921.

Harlow BL, Signorello LB, Hall JE, Dailey C, Komaroff AL (1998) Reproductive correlates of chronic fatigue syndrome. American Journal of Medicine 105(3A): 94S-99S.

Hollins Martin CJ (2008) Birth planning for midwives and mothers. British Journal of Midwifery 16 (9):583-587.

Hollins Martin CJ (2012) Personality disorder and mental health in the perinatal period. In Martin CR (ed) Perinatal mental health: a clinical guide (pp 43-57). M \& K Update, Keswick.

Hollins Martin CJ, Fleming V (2011) The Birth Satisfaction Scale (BSS). International Journal of Health Care Quality Assurance 24(2): 124-135.

Jason LA, Porter N, Hunnell J, Rademaker A, Richman JA (2011) CFS prevalence and risk factors over time. Journal of Health Psychology 16(3): 445446.

Jason LA, Taylor R, Wagner L (1995) Estimating rates of chronic fatigue syndrome from a community-based sample: a pilot study. American Journal of Community Psychology 23(4), 557-568.

Kaiser J (2011) Chronic Fatigue Syndrome. Studies point to possible contamination in XMRV findings. Science (New York.) 331(6013): 17.

Knightly R. (2008) Postnatal depression and unhappiness. British Journal of Midwifery 16(4): 258- 260. 
MacDonald KL, Osterholm MT, LeDell KH (1996) A case<en>control study to assess possible triggers and cofactors in chronic fatigue syndrome. American Journal of Medicine 100(5): 548-554.

Mayberry L, Gennaro S, Strange L, Williams M, De A (1999) Maternal fatigue: implications of second stage labor nursing care. Journal of Obstetric, Gynecologic and Neonatal Nursing 28, 175- 181.

Moses-Kolko EL, Roth EK (2004) Antepartum and postpartum depression: Healthy mom, healthy baby. Journal of the American Medical Women's Association 59(3): 181-91.

Murray D, Cox JL, Chapman G, Jones P (1995) Childbirth: life event or start of long-term difficulty? Further data from Stoke-on-Trent controlled study of postnatal depression. British Journal of Psychiatry 166: 595-600.

Njoku MG, Jason LA, Torres-Harding SR (2007) The prevalence of chronic fatigue syndrome in Nigeria. Journal of Health Psychology 12(3): 461-74.

O'Hara MW (2009) Postpartum depression: What we know. Journal of Clinical Psychology 65(12): 1258-1269.

Ostensen M, Villiger PM (2007). The remission of rheumatoid arthritis during pregnancy. Seminars in Immunopathology 29(2): 185-91.

Reeves WC, Jones JF, Maloney E. (2007) Prevalence of chronic fatigue syndrome in metropolitan, urban, and rural Georgia. Population Health Metrics 8(5):5.

Reyes M, Dobbins J, Mawle A (1996) Risk factors for chronic fatigue syndrome: a case<en>control study. Journal of Chronic Fatigue Syndrome 2: 17-32.

Richards JP (1990) Postnatal depression: a review of recent literature. British Journal of General Practice 40: 472-6.

Rosati P (2008) Chronic fatigue syndrome: implications for women and their healthcare providers during the childbearing years. Journal of Midwifery and Women's Health 53(4): 289-301.

Schacterle RS, Komaroff AL (2004) A comparison of pregnancies that occur before and after the onset of chronic fatigue syndrome. Archives of Internal Medicine 164(4): 401-404.

Schur E, Afari N, Goldberg J, Buchwald D, Sullivan PF (2007) Twin analyses of fatigue. Twin Research Human Genetics 10(5): 729-733.

Sinaii N, Cleary SD, Ballweg ML, Nieman LK, Stratton P (2002) High rates of autoimmune and endocrine disorders, fibromyalgia, chronic fatigue syndrome 
and atopic diseases among women with endometriosis: a survey analysis. Human Reproduction 17(10): 2715- 2724.

Smith MS, Buchwald DS, Bogart A, Goldberg J, Smith WR, Afari N (2010) Adolescent offspring of mothers with chronic fatigue syndrome. Journal of Adolescent Health 46(3):284-291.

Straus SE, Dale JK, Wright R, Metcalfe DD (1988) Allergy and the chronic fatigue syndrome. Journal of Allergy and Clinical Immunoloogy 81(5): 791-795.

Taillefer SS, Kirmayer LJ, Robbins JM, Lasry JC (2003) Correlates of illness worry in chronic fatigue syndrome. Journal of Psychosomatic Research 54(4): 331-337.

Walsh CM, Zainal NZ, Middleton SJ, Paykel ES (2001) A family history study of chronic fatigue syndrome. Psychiatric Genetics 11(3): 123-128. 